
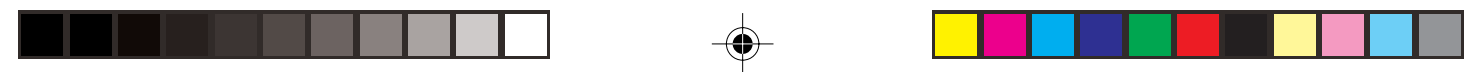

\title{
CHIRODERMA VILLOSUM (CHIROPTERA: PHYLLOSTOMIDAE) EN EL ESTADO DE YUCATÁN, MÉXICO.
}

\author{
JAVIER ENRIQUE SOSA-ESCALANTE, SILVIA HERNÁNDEZ Y \\ AUGUSTO SEGOVIA
}

\begin{abstract}
Departamento de Zoología, Facultad de Medicina Veterinaria y Zootecnia, Universidad Autónoma de Yucatán. Apartado postal: 4-116 Itzimná. Mérida, Yucatán, México.
\end{abstract}

Palabras clave: Chiroderma villosum, Chiroptera, Yucatán, México.

Se documenta por primera ocasión la presencia de Chiroderma villosum en Yucatán, lo que incrementa a 35 el número de especies de quirópteros con distribución en el Estado (Hernández et al., 1996).

Anteriormente la especies se había registrado como material subfósil, mediante la descripción de dos radios, una mandíbula y un fragmento de húmero en las grutas de Loltún, Yucatán (Arroyo-Cabrales y Álvarez, 1990). Ese registro y los registros en Campeche y Quintana Roo (Birney, et al., 1974; Jones et al., 1973), indicaron la posibilidad de la distribución de la especie en el Estado de Yucatán.

Nuestros ejemplares fueron capturados en la Reserva Estatal de Dzilam, ubicada al noreste de Yucatán, entre los municipios de Dzilam de Bravo (21²4’45” $\mathrm{N}$

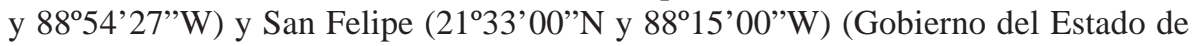
Yucatán, 1988). El clima corresponde al BS1 (h’) w” (x’), que representa el más húmedo de los climas cálidos secos, con una temperatura y precipitación media anual que fluctúa entre 24.7 y $26.4^{\circ} \mathrm{C}$ y 600 y $700 \mathrm{~mm}$, respectivamente. El área carece de corrientes superficiales permanentes y tiene una elevación de 4 metros sobre el nivel del mar.

La captura de los ejemplares se realizó con redes nylon de $30 \mathrm{~mm}$ de apertura de malla con $12.5 \mathrm{~m}$ de longitud por $2.4 \mathrm{~m}$ de ancho, instaladas en selva baja caducifolia con cenotes, selva baja caducifolia espinosa, manglar, vegetación de duna costera y pastizales inducidos con aguadas. Se colectaron un total de 7 individuos de C. villosum que están depositados en la Colección Zoológica Regional de la Universidad Autónoma de Yucatán (números de catálogo COZORE 25, 68, 105, 116, 139, 140, 141).

Las medidas somáticas (mm) y la masa corporal (g) de dos hembras (COZORE 25, 116) y dos machos (COZORE 68, 105), son respectivamente las siguientes: 
longitud total 68.0, 69.0, 67.5, 71.0; longitud de la pata 12.0, 11.9, 11.6, 12.0; longitud de la oreja 21.0, 17.7, 18.0, 20.0; longitud del antebrazo 46.0, 48.1, 48.0, 46.4; peso 24.5, 26.9, 24.3, 23.2. Las medidas craneales $(\mathrm{mm})$ de tres hembras (COZORE 25, 116, 140) y cuatro machos (COZORE 68, 105, 139, 141), son respectivamente las siguientes: longitud caja craneal 25.0, 24.4, 24.1, 24.0, 24.5, 24.0, 23.6; longitud condilobasal 23.0, 22.5, 21.5, 22.8, 22.4, 21.8, 21.8; longitud de la serie de dientes maxilares 9.3, 9.4, 8.8, 8.6, 8.7, 8.7, 9.0; ancho cigomático 16.1, 16.3, 15.6, 15.7, 15.6, 16.0, 15.2; ancho interorbital 3.5, 3.2, 4.3, 3.2, 3.2, 3.3, 4.5.

En septiembre de 1992 se capturó una hembra lactante y en abril de 1993 un macho inactivo (7.9 km NW Rancho San Salvador, km 2 de la carretera Yalsihom-

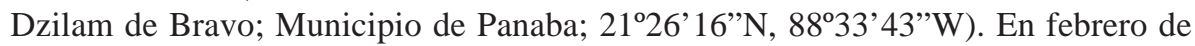
1993 se colectaron dos hembras, una lactante y una gestante con un embrión de $0.5 \mathrm{~g}$ en las primeras etapas de desarrollo (12.2 km SE rancho San José, km 20 de la carretera Panaba-Yalsihom; Municipio de Panaba; 2120’11”N, 88²3’31”W). En octubre de 1992 se colectó un macho con testículos escrotados con medidas de 6 x 4 mm (8.5 km N Rancho San Miguel, km 16.6 de la carretera Yalsihom-Dzilam de

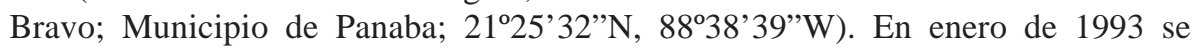
capturó otro macho con testículos escrotados, con medidas de 4 x 2.65 mm (5.6 km NE Rancho El Oasis, km 46.5 de la carretera Yalsihom-Dzilam de Bravo; Municipio

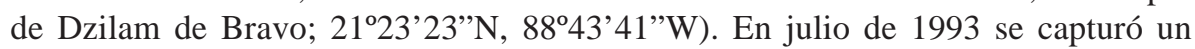
macho del cual no se obtuvo información reproductiva (11.5km N Rancho Sinkehuel, km 31.9 de la carretera Yalsihom-Dzilam de Bravo; Municipio de Dzilam de Bravo;

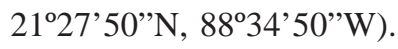

Estos ejemplares se capturaron con Artibeus intermedius, Artibeus jamaicensis, Dermanura phaeotis, Glossophaga soricina y Sturnira lilium. En otras localidades de México, estas especies también han sido registradas en asociación con C. villosum (Jones et al., 1973; Sánchez y Romero, 1995).

Nuestros registros constituyen los más norteños en la Península, ya que la localidad más cercana corresponde a Playa del Carmen, Quintana Roo, aproximadamente a $175 \mathrm{~km}$ de distancia de nuestras localidades (Birney et al., 1974). No existían registros de la especie en dunas costeras y humedales de México (Martínez et al., 1993). Es posible que estos sistemas sean usados por la especie para buscar alimento y la comunidad adyacente a la duna costera, que es principalmente el manglar le sirva como refugio. C. villosum ocupa hábitats similares con vegetación riparia en otras regiones de la Península (McCarthy, 1987).

Es probable que en el noreste de Yucatán, C. villosum presente dos periodos reproductivos; el primero de enero a marzo y el segundo de agosto a octubre. Esto confirma un patrón poliéstrico bimodal sugerido para el este de Quintana Roo y sur de Campeche, con picos de diciembre a marzo y a la mitad del verano (Jones et al., 1973; 
Navarro et al., 1990) y para el suroeste de Tabasco, con periodos de gestación de marzo a mayo y de agosto a octubre (Sánchez y Romero, 1995). En Chiapas y en regiones de América Central existe por lo menos un periodo reproductivo claro que va de marzo a mayo (Álvarez-Castañeda y Álvarez, 1991; Davis et al., 1964; Gardner et al., 1970; Jones et al., 1971). En las Antillas el periodo de gestación se presenta de junio a octubre (Goodwin y Greenhall, 1961).

Agradecemos a las autoridades de la Facultad de Medicina Veterinaria y Zootecnia de la Universidad Autónoma de Yucatán y al programa de Ecología Terrestre de la Secretaría de Educación Pública. A la Colección Nacional de Mamíferos del Instituto de Biología y a la Dirección General de Intercambio Académico de la Universidad Nacional Autónoma de México, por el apoyo brindado a través del Programa de Intercambio con la UADY. A. G. Correa, L. Brito, R. Novelo, L. Castillo, A. Zavala y P. García, por su colaboración en el trabajo de campo. V. Sánchez-Cordero, F. A. Cervantes y J. Arroyo-Cabrales proporcionaron valiosos comentarios a este manuscrito.

\section{LITERATURA CITADA}

Álvarez-Castañeda, S. T. y T. Álvarez. 1991. Los murciélagos de Chiapas. Instituto Politécnico Nacional, Escuela Nacional de Ciencias Biológicas, México, D. F.

Arroyo-Cabrales, J. y T. Álvarez. 1990. Restos óseos de murciélagos procedentes de las excavaciones en las grutas de Loltún. Instituto Nacional de Antropología e Historia, Serie Prehistoria, Colección Científica, 194: 5-103.

Birney, E. C., J. B. Bowles, R. M. Timm y S. L. Williams. 1974. Mammalian distributional records in Yucatan and Quintana Roo, with comments and reproduction, structure and status of peninsular populations. Occasional Papers, Bell Museum of Natural History, University of Minnesota, 13:1-25.

Davis, W., D. Carter y R. Pine. 1964. Noteworthy records of Mexican and Central American bats. Journal of Mammalogy, 45: 375-387.

Gobierno del Estado de Yucatán. 1998. Plan de manejo de la Reserva de Dzilam, Yucatán, México. Mérida, Yucatán.

Goodwin, G. y A. Greenhall. 1961. A review of the bats of Trinidad and Tobago. Bulletin of the American Museum of Natural History, 122: 195-302.

Hernández, S., V. Sánchez-Cordero, J. Sosa-Escalante y A. Segovia. 1996. Lista anotada de los mamíferos terrestres de la Reserva de Dzilam, Yucatán, México. Listados Faunísticos de México, Instituto de Biología, Universidad Nacional Autónoma de México, 7: 1-39.

Jones, K., J. Smith y H. Genoways. 1973. Annotated checklist of mammals of the Yucatan Peninsula, Mexico. I. Chiroptera. Occasional Papers of the Museum. Texas Tech University, 13: 1-31.

Jones, K., J. Smith y R. Turner. 1971. Noteworthy records of bats from Nicaragua, with checklist of the chiropteran fauna of the country. Occasional Papers of the Museum of Natural History, University of Kansas, 2: 1-35. 
Martínez, M., P. Moreno y S. Castillo.1993. Biodiversidad costera: Playas y dunas. Pp. 160181, en: Biodiversidad marina y costera de México (S. Salazar y N. González, eds.). CONABIO, CIQRO, México, D. F.

McCarthy, T. 1987. Distributional records of bats from the Caribbean lowlands of Belize and adjacent Guatemala and Mexico. Pp. 157-172, en: Studies in Neotropical Mammalogy: Essays in honor of Philip Hershkovitz (B. D. Patterson y R. M. Timm, eds.). Fieldiana Zoology, New Series, 39: 1-286.

Navarro, D., T. Jiménez y J. Juárez. 1990. Los mamíferos de Quintana Roo. Pp. 371-450, en: Diversidad Biológica en la Reserva de la Biosfera de Sian Ka'an Quintana Roo, México (D. Navarro y J. Robinson, eds.). Centro de Investigaciones de Quintana Roo, Chetumal, Quintana Roo.

Sánchez, C. y L. Romero. 1995. Murciélagos de Tabasco y Campeche: Una propuesta para su conservación. Cuadernos del Instituto de Biología, Universidad Nacional Autónoma de México, México D. F. 